\title{
Discussion on the Power of a Dream in Sports Movie 《Mr.3000》
}

\author{
Zhou Chuan \\ Sports Department of University of Electronic Science and Technology of China, Chengdu, Sichuan, China
}

\begin{abstract}
The "Mr.3000" is directed by Charles stone distinctive visual style and colors are used to deduce a new baseball comedy movie. Film tells the story of professional baseball player Stan Ross to pursue their dreams - enter the list of baseball celebrity in touching story appears to have to fight for. Ten years ago, when Ross finished to ensure the 3000th on the list of baseball celebrity hits after, immediately decided to retire, then start the business plan, "Mr. 3000" in the final certification rose into celebrity list after ten years, his three hits record is considered null and void, he had to restart the bat, complete the final three hits. Lines clear film storyline simple but profound meaning, the middle-aged man dream to change more than 40 years of the life attitude of hesitation and resolve performance incisively and vividly, with unique emotional and visual angle is an illustration of the power of a dream, and through the form of exciting and funny and make people fully enjoy the movie art special charm.
\end{abstract}

Key words — baseball movies, dreams, positive energy, academy of motion picture arts

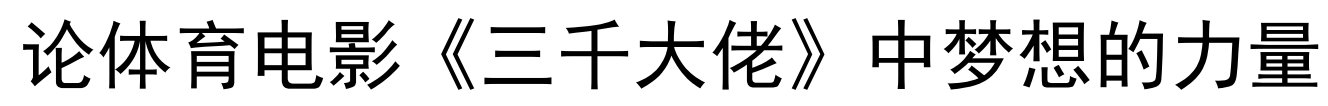

周川

电子科技大学体育部，成都，四川，中国

摘 要 《三千大佬》是由导演查理斯·斯通运用与众不同的视觉风格和色彩来演绎的一部全新的棒球喜剧电影。影片讲述了职业棒 球运动员斯坦・罗斯为了追逐自己的梦想一进入 “棒球名人榜”而在不惑之年不得不重新奋争的感人故事。十年前, 当罗斯完成确保进入 “棒球名人榜”的第 3000 个安打之后，便立即决定退役，随后开始了“Mr.3000”的商业计划，而在十年后最终认证罗斯进入名人榜时， 他的 3 次安打记录被判定无效，他不得不重操球棒，完成最后的 3 次安打。影片故事情节简单主线明确却寓意深刻，将步入中年的男 人为了梦想改变四十多年以来人生态度的彷得与决心表现的淋漓尽致, 用独特的情感角度和视觉角度诠释了梦想的力量, 并通过激动 人心而滑稽的手法表现出来, 使人们充分享受电影艺术非凡的魅力。

关键词 棒球影片，梦想，正能量，电影艺术

\section{1. 引言}

曾几何时，电影与体育结合就免不了招来非议，所以 印象中经典的体育影片实在是少之又少。现在, 人们倒是 想开了, 谁说体育影片就只能正儿八经? 于是柳暗花明又 一村, 加上了喜剧浪漫等各种调料的体育影片再度成为好 莱坞最受欢迎的题材之一 $[1]$ 。

而要问体育运动里什么项目在美国最为盛行, 除了橄 榄球和篮球, 棒球也能让美国人疯狂。《三千大佬》(原片 名 《Mr.3000》, 又译为《安打先生》) 就正好迎合了美国人 的胃口，不仅招来了一批黑人明星疯狂搞笑，也直接挑战
美国人顶礼膜拜的 3000 安打, 喜剧效果立竿见影。然而吸 引人的不仅仅是精彩的比赛情节或者让人捧腹的笑料, 其 中蕴涵的励志与哲理也是打动人的主要因素。

2. 《三千大佬》: 故事主线明确, 拍摄真实质感 2004 年，黑人体育喜剧电影《三千大佬》是导演查理 斯-斯通继连续拍摄了 2 部黑人题材电影：描写黑人街区生 活的《全款交收》(Paid in Full)和讲述黑人学校生活的《乐 鼓队》(Drumline)之后的又一力作, 这 3 部电影已经完全证 明了查理斯・斯通对黑人题材电影把握的功底以及将剧情 和幽默揉合在一起的非凡创造力。 
3000 次安打是棒球运动罕见的记录, 迄今为止只有 25 人创此佳绩。每个运动员都希望用杰出的竞技表现诠释自 己的职业生涯。由伯尼·麦克饰演的影片主人公斯坦・罗斯的 梦想就是成为第 26 个人。斯坦 $\cdot$ 罗斯长着一张大嘴, 十年前, 他是一个极其自负, 以自我为中心的人, 从不对着镜头微 笑, 从不会讨好媒体, 也不会给任何人签名, 所以人们不 喜欢他但又忍不住爱他, 只因为他是一名极具天赋的职业 棒球运动员。

幸运的他命中注定要完成自己的梦想。在他 37 岁那年, 在职业大联盟上完成了确保他进入棒球名人榜的第 3000 次 安打。然而, 出乎所有人的预料, 正值事业颠峰的他却决 定立即退出比赛, 中途退赛抛弃了处于困境中的球队和队 友。在完成这个骄人战绩之后, 他因此获得“三千记先生” 的绰号, 他的“21”号球衣也记载着他曾经的光荣。但是因 为糟糕的人际关系十年间一直未进入“棒球名人堂”, 不过 这并不妨碍罗斯凭借“三千记先生”的威名拓展自己的事 业, 他旗下以“3000”命名的公司业务蒸蒸日上。

然而真正的高潮才刚刚开始。就在他几乎遗忘棒球的 时候, 两个好事的记者发现之前的安打记录有误, 斯坦・罗 斯为密尔沃基-布鲁尔队效力时所完成的 3 记安打被判定无 效, 他真正完成的应该是 2997, 如果这样的话, 罗斯的名 人堂资格、“三千记先生”的赫赫声名和那些依靠这些带动 的事业都将不复存在, 这个数字是世界上对他最重要的东 西, 进入 “棒球名人堂” 的梦想也寄托于此, 为了梦想, 他 只有一种选择, 就是戒除不良嗜好, 恢复训练, 重操球棒, 回到密尔沃基-布鲁尔球队, 补齐 3000 次安打, 影片所有的 一切都围绕这个主线展开。

如果是 10 年前的他, 罗斯可以用 20 分钟完成所有的 一切, 然而现在他已经 47 岁, 荒诞生活让他的身体和意志 都变得虚弱, 没有人相信这个全身肥肉的老家伙能打出安 打。为了进入 “棒球名人堂”的梦想, 47 岁的罗斯不得不从 一垒开始打起, 失常的表现一度遭到非议, 很快他变成了 新闻的笑柄和球队的祸害, 罗斯也一度失落, 只有体育记 者西蒙丝(安吉拉・贝赛特饰)还对他的实力深信不疑。在球 队的安排下及西蒙丝的鼓励下, 罗斯开始了漫长的恢复练 习, 在接下来的比赛中, 罗斯突然发现自己对棒球的热爱 并未完全泯灭, 而且球迷们依旧那么爱他。他也在比赛中 逐渐认识到自己从前骄傲自大的无知, 学会了如何将自己 融入到整个团队之中, 学会了如何用集体的力量来克服自 己的薄弱。随着赛季的延续, 罗斯逐渐找回了当初的感觉, 他开始意识到这并不只是数字的游戏, 狂妄自大的罗斯终 于体会到了团队合作的乐趣, 学会用道德规范约束自己, 甚至坠入了爱河。现在, 那三记安打好像越来越近了, 梦 想也越来越近了。
然而在影片的最后, 罗斯为了球队的胜利并未完成第 3000 次安打, 因此他职业生涯的安打锁定在 2999 次。虽然 罗斯失去了进入 “棒球名人堂”的资格, 但是他为自己球队 的胜利牺牲自我的精神, 赢得了球迷们的热爱和尊重, 感 动了评委, 破格进入 “棒球名人堂”, 罗斯如愿完成了自己 梦㝥以求的梦想。

整部影片对棒球比赛真实性的刻画十分到位, 体现出 棒球运动真实、精彩的一面。影片中的演员就像职业运动 员一般能够击球、接球和跑垒, 影片多次将镜头在球场和 球队粉丝之间切换, 让人错觉以为不是在看电影, 而是在 观看一场惊心动魄的棒球直播比赛。

同时, 导演查理斯・斯通运用与众不同的视觉风格和色 彩来演绎这部全新的棒球电影。让观众体会到棒球运动的 真实质感, 感受到球棒的大力冲击, 用真实标新立异。影 片中采用长焦镜头拍摄捕捉棒球比赛场景的方法不仅使视 野开阔, 而且球场的现场感十分强烈, 再加上特写镜头的 运用, 造就了画面真实感和情感上的冲击力。当球队比赛 时, 我们似乎能清晰听到自己的心跳声。另外, 在颜色上 也是独具匠心, 比如影片中早期棒球比赛中的颜色非常鲜 艳惹眼, 而在罗斯退休后, 比赛的颜色在蓝色色调中柔和 暗淡下来。

\section{3. 《三千大佬》正能量一: 改变自我, 向梦想出发}

每一个人都有自己最诚挚的梦想, 罗斯的梦想就是要 让他的名字记入 “棒球名人堂”。为此, 他坚持不解的打了 3000 次安打, 而后毅然退役。然而世事弄人, 退役多年的 罗斯在不惑之年突遭变故, 出乎意料的推翻了引以为荣的 数字, 而此时罗斯离梦想的距离只有 3 次安打的距离。人 到中年依然可以改变, 但是这改变相当艰难。一个正值事 业颠峰的人突然堕入常人的境地, 复杂的心情难以形容。 这个数字是世界上对他最重要的东西, 他突然失去了 3000 次安打记录, 不得不重新审视过去和将来, 在过去他认为 所要做的一切就是击球, 天赋和狂妄将他变成了怪物, 他 必须克服自我, 做出改变才能到达梦想的彼岸。他必须学 会作一个了不起的人, 而不只是一个了不起的运动员。

影片一开始就将罗斯的缺点一一暴露在观众面前。罗 斯有着阳光般的笑脸, 也有着招人怨恨的一面; 他衣冠楚 楚、魅力迷人, 却有些被自私蒙蔽双眼; 他是了不起的运 动员, 可与自己周围的世界格格不入。最让人印象深刻的 是在他完成第 3000 次安打后球飞出场地飞向观众席的时 候, 球落在了一个小孩的手里。而此时此刻, 他眼中只有 他的 3000 安打, 只有他的球, 罗斯径直走下球场, 直接奔 小孩走去。大明星向观众席走来, 赢得大家一片欢呼声, 然而他的脸上没有一丝的微笑, 紧绷着脸大步走到拿球小 
孩面前。此时小孩的父亲特别兴奋的要拥抱罗斯, 罗斯一 点也不理会球迷的热情, 冷生生的说, 球在哪里? ! 小孩 的父亲很是尴尬, 打圆场说, 可以拿你的帽子或是签名来 交换一下。本来这对于罗斯来说举手之劳的事情, 而他的 眼中只有 3000 安打, 只有他的球, 其他一切都与他无关。 最后, 罗斯硬生生从小孩的手中夺过球后离开球场, 引来 一片嘘声。这充分暴露了罗斯以自我为中心, 心中毫无他 人的自私性格。影片还从其他两个角度突出了罗斯自私的 性格缺陷。第一个是罗斯与球队及队友之间的关系。在罗 斯退役前的最后一场比赛中, 罗斯所在球队一直在输球, 急需罗斯带领球队赢得比赛。教练和球迷也对他寄予厚望。 而罗斯却在打完他人生中的 3000 安打后, 立即宣布退赛, 将球队和队友们抛弃, 并且在新闻发布会将队友贬的一文 不值。当记者问及此时退赛会不会影响他的球队时, 他竟 然很气愤的说, 这不是他的球队, 他与这个球队无关。教 练及队友听到这些是何等的伤心, 在他们朝夕相伴, 一起 并肩作战的大明星心中, 他们没有任何价值可言。此时镜 头的画面给了教练一个特写, 脸上写满了无奈和厌恶。鉴 于罗斯的如此行径, 在多年以后举行的“纪念罗斯 3000 安 打” 的活动中, 到场的队友胗胗无几, 而到场的队友在发表 演讲谈及对罗斯的印象中, 也没有一人能说出除了打球技 艺高超之外的其他优点, 大家都是含糊其辞草草了事。罗 斯眼中没有他人, 他人眼中自然就没有罗斯。第二个侧面 反映罗斯自私性格的是在他退役后开办公司, 虽然事业在 他个人名望的带动下蒸蒸日上, 身边的朋友却越来越少。 他常以 3000 安打的辉煌战绩向人炫耀, 在胜利和荣耀的顶 端让他开始变得以自我为中心, 自大自私和蛮不讲理的性 格伤害了周围的朋友, 纷纷离他而去。

罗斯认为不论过程如何, 结果意味着一切, 不管他做 了什么, 他总会因数字 3000 而自我感觉良好, 于是影片中 导演剥去他自大傲慢自私的外表, 让他身处无能为力的境 地, 强迫他面对挑战。为了最后的 3 个安打, 为了进入“棒 球名人堂”的梦想, 罗斯的人生轨迹也发生了重大改变, 自 大自私的性格也在争取最后的 3 个安打中随之东去。

开始罗斯自信自己能够轻松打出 3 个安打, 而事实上 47 岁的罗斯反应速度已经远远落后于巅峰时期。斯坦人已 中年, 做几个仰卧起坐俯卧撑都勉勉强强, 更别提越来越 大脾气的他根本没有容忍之量, 鉴于他之前的表现, 以前 的队友也一直对他耿耿于怀。斯坦走到了人生的十字路口, 坚持还是放弃? 此时在斯坦复出之际, 女主角西蒙斯也出 场了。作为感化斯坦・罗斯并对其命运至关重要的女主角, 西蒙斯的角色在整部电影中起到了灵魂作用。一个眼光独 到的体育记者, 坚韧而不失妩媚, 头脑机智聪慧, 与罗斯 的感情微妙复杂, 更具有感化顽固的罗斯的人格魅力。在
所有人嘲笑讽刺他的时候, 西蒙斯坚定的认为斯坦是他心 中最棒的。一定能够打出最后的三个安打。在被所有人的 打击下, 也许这是斯坦身陷困境时的最后一根稻草, 给了 斯坦精神上的动力。西蒙斯动摇了罗斯的世界, 使一个男 人时值中年才第一次学会去爱。在西蒙斯的鼓励下, 罗斯 走向了漫长的复出之路。

在比赛中, 罗斯再也不是高高在上, 再也不能轻松的 打出安打, 他现在只是一个普普通通的棒球选手, 一切都 要从零做起。这也使他重新认识了棒球这项运动, 不只是 他一个人的棒球, 而是一个团队的棒球。一次又一次的失 败促使罗斯更加注重团队的力量, 更加注重别人的感受。 首先, 罗斯认识到每个人在运动场上都有他的存在价值, 不能因为自己技艺高超而对其他人虽之以鼻。影片中罗斯 在被别人差辱一番后, 心中五味杂陈, 联想起自己以前就 是这样对待自己的队友, 让自己的队友难堪, 罗斯心中愧 疚不已。对待别人的差辱他没有任何资格反驳, 只好默默 走开。从此, 罗斯再也没有随随便便谩骂过队友, 让他懂 得了尊重别人, 别人才会尊重自己。其次, 罗斯学会了分 享。罗斯给人的印象总是自大自负, 而在追求最后的 3 个 安打中, 罗斯不断地与队友分享自己的打球技巧与心得, 帮助队友进步。影片中在一次比赛中, 球队一直未能接住 对方的球, 不断的出局, 罗斯凭借多年的经验发现了对手 胳膊肘拐进去扔曲线球的秘密, 并将对方暴露的投球路线 奉献出来, 与队友一起分享。这在以前是不可能发生的事 情, 教练也很奇怪的盯着罗斯, 感觉罗斯像是变了一个人 一样。队友在他的指导下, 打出了一个漂亮的本垒, 并取 得了本次比赛的胜利。最后, 罗斯的形象在他人生中的最 后一次比赛中完全蜕化。彻底从一个自私的人学会了无私。 影片中在罗斯再次退役前的最后一次比赛中, 万众瞩目, 比赛进行到了白热化, 进行到下半场, 双方比分依然是 0 : 0 , 此时罗斯离 3000 安打只差一个。在一次上垒中, 罗斯 被裁判判罚出局。罗斯很是失落, 若是以前罗斯肯定会过 去脾气暴躁的大骂裁判, 然而他却很冷静的接受了这一判 罚。此时, 另一个人气冲冲的冲上球场与裁判理论, 他就 是罗斯的教练。罗斯的蜕变, 教练一直看在眼里, 他以一 个年长者的身份一直在保护他, 期待罗斯更好的变现。这 次理论, 也让罗斯认识到自己在教练心中依然是那个技艺 高超的棒球小子, 没有因为自己以前的过错一直怀恨在心。 在比赛的最后, 罗斯面临着两难的选择, 要么自己打 3000 安打让球队输球, 要么自己放弃最后一次机会让球队赢球。 此时此刻, 罗斯百感交集, 自己一直以来的奋斗都是为了 补齐最后的 3 个安打完成自己的梦想。而这之间发生的事 情, 让罗斯再也不能像以前一样随便抛弃球队, 抛弃队友。 他回忆起了种种以前的事情, 最后自己故意出局, 为队友 
得分创造条件, 自己也成为了“2999 先生”。与之前形成鲜 明对比, 最后的那个球, 他没有收藏起来, 而是大方的扔 向了观众席。看到这里, 我们不免有些感动。常言道, 山 难移性难改, 一个中年人改变自己四十多年的性格是多么 的不容易。

人生中的很多际遇只在一念之间, 倘若错过, 便空留 遗憾[2]。运动员的职业生涯中, 或许再多一次可能便可登 峰造极, 些许人就因这一次便碌碌平庸, 前功尽弃。罗斯 为了这一次付出了艰辛的汗水, 从中更懂得了人生的意义, 改变了自己的世界观。虽然没有完成最后的 3 个安打, 罗 斯的无私的精神打动了每一个人, 如愿的完成了自己的梦 想—进入“棒球名人堂”。

\section{4. 《三千大佬》正能量二: 感悟人生, 感染他人}

一个人的成功不是真正的成功, 团队的成功才是最大 的成功。之前, 罗斯一直是一个了不起的运动员, 而他却 不是一个了不起的人。他的个人成功来源于自己的天赋和 努力。在生命的历程中, 从未感受过生命的意义所在, 只 是一个自私自利活在自己世界的一个人[3]。在经过这次人 生重大转折之后, 罗斯开始品味这个世界, 眼中不仅仅有 自己, 更有自己要爱的人和那些爱自己的人。之前, 罗斯 从不承认自己对西蒙斯的感觉, 因为他一向自如的周旋于 女人中间, 不想让自己受到丝毫约束, 但随着爱情火花的 产生, 罗斯意识到西蒙斯是命运安排给自己的。自己再不 能像以前那样毫无责任感, 没有起码的人生操守。生命中 的点点滴滴都值得他去珍惜, 用心去感受。

在影片中罗斯同样为年轻球员树立应有的榜样, 用自 己的实际行动去感染他人。影片中的另一个配角是罗斯的 队友号称 “田纳西霸王龙” 的潘贝克, 潘贝克就像是罗斯年 轻时的影子, 球技高超而又自大自负傲慢无礼, 没有一丝 的团队精神。罗斯正是从他身上不断地反思自己以前的所 作所为, 同时也不愿意看到潘贝克重蹈自己的覆辙。当罗
斯刚刚复出进入球队时, 潘贝克对这个老头不屑一顾, 时 不时讽刺挖苦他。罗斯一忍再忍, 并没有产生怨恨心理。 而后在比赛中, 罗斯不断地找机会把自己的比赛经验教授 与他, 因为他知道自己再次退役后, 潘贝克将是球队的核 心。在影片中罗斯表现出了极度的耐心和一位长者的风范, 以自己的切身体会开导潘贝克, 在罗斯的不断感召下, 潘 贝克终于认识到自己的错误, 重新树立起自己人生价值观 念, 带领球队冲向胜利。

一个成功的人带领自己的团队走向成功才是真正的成 功, 在影片中, 罗斯在他所经历的改变中不断地反思自己, 在追求梦想的过程中感悟人生的意义与美好, 用积极的人 生态度感染他人, 值得我们每一个人学习 [4]。

\section{5. 结语}

影片来源于真实生活的喜剧情节, 虽然诙谐却不乏辛酸。 在影片中罗斯不仅仅是体育明星, 还是一向顽固不化而最 终却只能妥协的滑稽男人, 罗斯的形象也被刻画得温暖而 有趣。罗斯形象不仅仅是当代体坛超级巨星的缩影, 更涵 盖了顽固不愿改变而不得不屈从于现实的所有人。《三千大 佬》中罗斯也给每一个追求梦想的人以启迪一一向着梦想 出发, 勇敢向前。

\section{参考文献(References)}

[1] H. F. Cao, "On the acceptance dimension of the movie audience," Master's degree thesis of Qufu Normal University, 2003.

[2] L. Zhu, "Digital technology influence the Hollywood film visual effects," Journal of Beijing Film Academy, no. 5, pp. 22-28, 2005.

[3] L. L. Jia, "The cultural responsibility of national film," Contemporary Cinema, no. 9, pp. 41-43, 2009.

[4] X. G. Chen, "2009: Chinese movie art and industry memorandum," Arts Criticism, no. 2, pp. 5-13, 2010. 